\title{
Bipolar Electrode Array Embedded in a Polymer Light-Emitting Electrochemical Cell
}

Jun Gao1, Shulun Chen1, Faleh AlTal1, Shiyu Hu1, Laurent Bouffier2 and Guillaume Wantz3

1Department of Physics, Engineering Physics and Astronomy, Queen's University, Kingston, Ontario, K7L 3N6, Canada

2Univ. Bordeaux, ISM, CNRS, UMR 5255, Bordeaux INP, F-33400 Talence, France

3Univ. Bordeaux, IMS, CNRS, UMR 5218, Bordeaux INP, F-33405 Talence, France

\begin{abstract}
A linear array of aluminum discs is deposited between the driving electrodes of an extremely large planar polymer light-emitting electrochemical cell (PLEC). The planar PLEC is then operated at a constant bias voltage of $100 \mathrm{~V}$. This promotes in situ electrochemical doping of the luminescent polymer from both the driving electrodes and the aluminum discs. These aluminum discs function as discrete bipolar electrodes (BPEs) that can drive redox reactions at their extremities. Time-lapse fluorescence imaging reveals that $\mathrm{p}$ - and $\mathrm{n}$-doping originated from neighboring BPEs can interact to form multiple light-emitting $p-n$ junctions in series. This provides a direct evidence of the working principle of bulk homojunction PLECs. The propagation of $\mathrm{p}$-doping is faster from the BPEs than from the positive driving electrode due to electric field enhancement at the extremities of BPEs. The near simultaneous formation of multiple light-emitting p-n junctions in series causes a sharp increase in cell current. This indicates that the region containing a BPE is much more conductive than the rest of the planar cell despite the latter's greater width. The p-and n-doping originating from the BPEs are initially
\end{abstract}


highly confined. Significant expansion and divergence of doping occurred when the region containing the BPE array became more conductive. The shape and direction of expanded doping strongly suggest that the multiple light-emitting p-n junctions, formed between and connected by the array of metal BPEs, have functioned as a single rod-shaped BPE. This represents a new type of BPE that is formed in situ and a combination of metal, doped polymers and forward-biased p-n junctions connected in series. 


\section{Introduction}

Bipolar electrochemistry exploits the wireless nature of bipolar electrodes (BPEs) for applications that are either inconvenient or impossible to achieve with conventional wired electrodes. For example, millions of micro-BPEs can be dispersed in an electrolyte solution and remotely addressed to generate electrochemiluminescence (ECL) in the bulk.1-2 Janus objects can also be synthesized in bulk with dispersed carbon tubes or carbon beads BPEs.3-4 Moreover, BPEs suspended in a liquid electrolyte can act as micro-swimmers when propelled by bubbles generated asymmetrically at the poles.5 Conceptually, a BPE is a floating conductor that can simultaneously drive reduction and oxidation reactions when polarized in an electrochemical cell.6-10 The BPE potential is not fixed to that of the driving (feeder) electrode. The redox reactions at the BPE surface are therefore controlled by the potential difference between the BPE and the surrounding electrolyte solution.

A solid-state analog of an electrochemical cell with dispersed micro-BPEs is the polymer "bulk homojunction" light-emitting electrochemical cell.11-12 A light-emitting electrochemical cell is a thin-film device made from mixed ionic/electronic conductors.13-19 Such a polymer lightemitting electrochemical cell (PLEC) was originally developed as an alternative to the polymer light-emitting diode (PLED). In both PLED and PLEC, electroluminescence (EL) occurs when injected electrons and holes recombine radiatively in the luminescent polymer. The active layer of a PLEC, however, also contains mobile ions that function as counterions to compensate the injected electronic charges. In operation, the luminescent polymer film in a PLEC is electrochemically p- and n-doped in situ. And a narrow light-emitting p-n junction is eventually formed when the dynamic doping fronts meet. 
Adding dispersed indium tin oxide or metal particles to the PLEC film dramatically alters the emission profile of the cell.11-12 Instead of a single light-emitting junction with a narrow depletion width and emission zone that occupies less than $1 \%$ of the entire cell area, thousands of point-like emitting junctions that account for over $40 \%$ of the cell area are formed between the driving electrodes. The conductive particles function as tiny BPEs from which doping are induced wirelessly. It is postulated that point-like light-emitting $p$-n junctions are formed between neighboring BPEs when opposite doping fronts make contact.

A “bulk homojunction" PLEC exhibits orders-of-magnitude increase in light output (vs. a single-junction PLEC of the same size) and a prompt turn-on response.11-12 The fast response time is, in part, due to the fact that the doping fronts only need to travel across the average distance between the BPE particles, as opposed to the entire distance between the driving electrodes, to form a light-emitting junction. However, the fast response time and small junction size also made it difficult to visualize the dynamic, collective doping process from multiple BPEs. In particular, it is unclear how exactly doping from neighboring BPEs interacts to form multiple light-emitting junctions. Since bulk homojunction LECs also exhibit a giant open-circuit voltage (Voc) when operated as a photovoltaic cell, it is postulated that multiple p-n junctions have formed in series via the BPE particles to give rise to a giant Voc that is much higher that the band gap of the luminescent polymer.11 This, however, also needs to be confirmed.

In this study, PLECs are fabricated with a single column of BPE discs embedded between the driving electrodes. The size and separation of the BPEs are chosen so that the collective behaviors of the BPE doping can be visualized using time-lapse fluorescence imaging. By carefully analyzing the doping pattern and cell current, we show that a linear BPE array allows for the simultaneous formation of a linear array of light-emitting $p-n$ junctions in series. This 
causes a measurable increase in cell current. The presence of a BPE array not only shortens the distance over which the doping fronts must propagate to form a junction, but also leads to a faster doping propagation speed when compared to the flat driving electrode. Single BPE arrays have already been demonstrated for high throughput, multiplexed detection or screening of biomolecules20-22 and catalysts23-25. In addition, 2D BPE arrays have been used to generate ECL or to functionalize graphene wirelessly.26-27 The current study shows that a BPE array is also a very useful tool to study the fundamental bipolar electrochemical processes. This is a major understanding since the principles of bipolar electrochemistry in a PLEC with embedded bipolar electrode were only established recently.28

\section{Experimental Methods}

In this study, we fabricated planar PLECs with an interelectrode gap of $10.38 \mathrm{~mm}$. Here, planar (vs. sandwich) implies that the driving electrodes are coated on the same surface of the active layer. The active layer between the driving electrodes is fully exposed. The extremely large planar PLECs, with a gap size of more than $10 \mathrm{~mm}$, are ideally suited for visualizing the doping processes via fluorescence imaging.29 The active layer of PLECs consists of three compounds. The luminescent polymer, poly[5-(2-ethylhexyloxy)-2-methoxy-1, 4-phenylene vinylene], MEH-PPV, is sourced from OLEDKing Optoelectronic Materials Ltd, China with a molecular weight of $\mathrm{Mw}=3.3 \times 105$ and a polydispersity index of 1.4 . The electrolyte polymer, polyethylene oxide $(\mathrm{PEO}, \mathrm{Mw}=2 \mathrm{M})$, and the potassium triflate $(\mathrm{KTf}, 98 \%)$ salt, were purchased from Sigma Aldrich and used as received. Cyclohexanone solutions of MEH-PPV and PEO:KTf were mixed to create a casting solution of MEH-PPV:PEO:KTf at a weight ratio of 1:1.3:0.25. $100 \mu \mathrm{l}$ of the solution was dispensed onto square glass or sapphire substrate and spun at $2000 \mathrm{rpm}$. The resulting polymer film had a thickness of $200 \pm 10 \mathrm{~nm}$, as determined with an 
Ambios optical profiler. The polymer film was dried at $50^{\circ} \mathrm{C}$ overnight to remove any residual solvent. The gold and aluminum driving electrodes and the aluminum BPE array are deposited by thermal deposition through a pair of shadow masks. The thickness of the gold and aluminum films are $60 \mathrm{~nm}$ and $100 \mathrm{~nm}$, respectively. A schematic of the finished planar PLEC is shown in Figure 1 (a). The device processing steps were carried out in a nitrogen-filled glove box/evaporator system to prevent exposure to oxygen and water.

The finished PLECs were placed in a sealed glass vial and transferred into a Janis ST-500 micro-manipulated cryogenic probe station for testing. A small amount of silver thermal paste was applied to the gold plated sample stage to improve thermal contact with the glass substrate and to provide a non-reflective background for imaging. The probe station chamber was then sealed and evacuated to a pressure of approximately $5 \times 10-4$ torr with an oil-free turbo-pump. Gold and tungsten probes of $100 \mu \mathrm{m}$ tip radius were brought into contact with the driving electrodes for testing. A Labview-controlled Keithley 237 high-voltage source measurement unit was used to source voltage bias to the probes and simultaneously measure the resulting current. The planar PLECs were imaged with a computer-controlled Nikon D300 digital SLR camera equipped with a Tamron $90 \mathrm{~mm} \mathrm{1:1} \mathrm{macro-lens.} \mathrm{A} \mathrm{UV} \mathrm{ring} \mathrm{lamp} \mathrm{provided} \mathrm{illumination} \mathrm{to} \mathrm{the}$ polymer film through the quartz window of the sample chamber. The temperature of the sample stage/planar LEC was controlled with a CryoCon temperature controller.

\section{Results and Discussion}

As shown in Figure 1 (a), the planar cell (Cell 1) has a gap size of $10.38 \mathrm{~mm}$ across the inner edges of the gold and aluminum driving electrodes. The BPE array, consisting of eight aluminum discs, is located on the right side of the cell but still well within the confines of the 
driving electrodes. The BPE array, therefore, is in an open configuration within the electrochemical cell. With the cell heated to $360 \mathrm{~K}$, a $100 \mathrm{~V}$ bias was applied across the driving electrodes. Figure 1 (b) shows the cell current as a function of time. The general trend in cell current is consistent with previous observations in large planar PLECs.29-30 The presence of a BPE array, however, has an observable effect on cell current that is absent in PLECs without a BPE array. This will be elaborated below.

Figure 1 (c) and Figure 1 (d) shows the time-lapse fluorescence images of Cell 1 in two locations, as depicted by the white rectangles in Figure 1 (a). Figure 1 (c) shows region L on the left away from the BPE array. Significant PL quenching occurred in the polymer film next to the gold and aluminum electrodes. The PL quenching is caused by in situ electrochemical doping of MEH-PPV in the mixed conductor polymer film. The dynamic electrochemical doping process in a planar PLEC has already been extensively studied via fluorescence imaging.29-31 In Cell 1 the dark $\mathrm{p}$-doped region and the lighter $\mathrm{n}$-doped region made full contact at about $\mathrm{t}=470 \mathrm{~s}$. The contact leads to the formation of a p-n junction, which is under forward bias, and the onset of electronic current that soon dominates the cell current. The formation of a $p-n$ junction in a planar PLEC is always accompanied by an accelerated increase, or turn-on, in cell current.29-31

Figure 1 (b) also reveals a much earlier turn-on in cell current at $\mathrm{t} \sim 35 \mathrm{~s}$. A more detailed view of this early turn-on will be shown in Figure 4 for Cell 2. This early turn-on in current is caused by the junction formation along the BPE array, long before a junction is formed in the rest of the device. As shown in Figure 1 (d), in situ electrochemical doping from the BPEs is observed early in the first image taken after the voltage bias was applied at $t=5 \mathrm{~s}$. Initially, only p-doping, propagating downward from the BPEs, is visible. Subsequently, sharp n-doping can be seen from $t=20 \mathrm{~s}$. At $\mathrm{t}=35 \mathrm{~s}$, the tips of the finger-like $\mathrm{p}$ - and $\mathrm{n}$-doped regions from the BPEs 
have made full contact to form multiple $p-n$ junctions in series. At $t=50 \mathrm{~s}$, the $\mathrm{p}$ - $\mathrm{n}$ junctions emit visible light against the strong background PL of the LEC film.

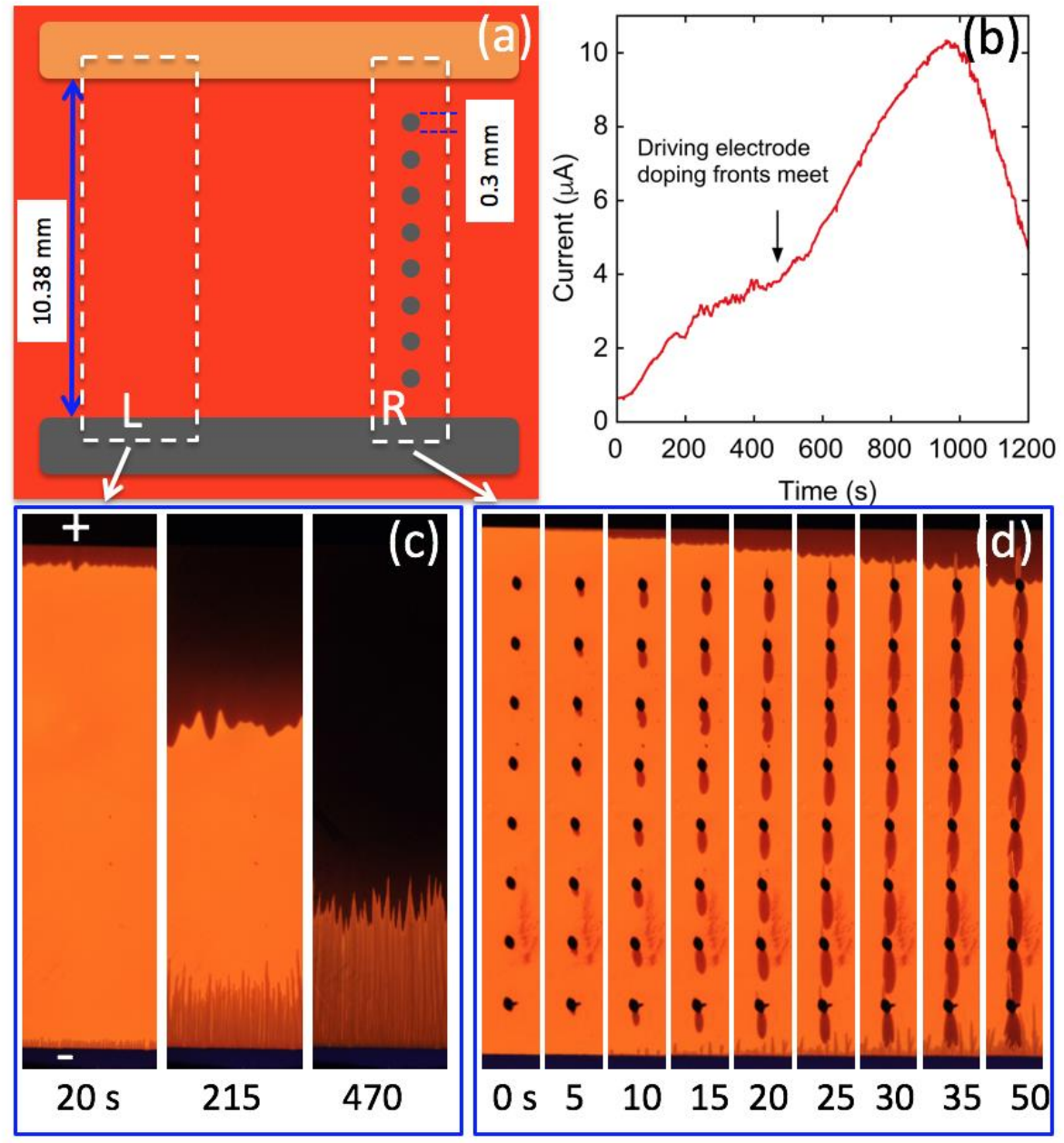

Figure 1. Cell 1-(a) Schematic of a planar PLEC containing an array of aluminum disc BPEs deposited on top of the polymer film, in a region denoted " $R$ ". " $L$ " denotes a region away from region $R$ and is unaffected by the presence of a BPE array. (b) Cell current as a function of elapsed time after a $100 \mathrm{~V}$ bias voltage was applied to the top gold (+) and bottom aluminum (-) driving electrodes. (c) Time-lapsed fluorescence images of region L. (d) Time-lapsed fluorescence images of region L. The numbers underneath the images in (c) and (d) denote elapsed time in seconds. The PLEC was submitted to UV illumination and heated to $360 \mathrm{~K}$ under vacuum. 
It is a significant observation that the formation of multiple $\mathrm{p}-\mathrm{n}$ junctions in series through the BPE array has caused a marked change to the overall cell current. The turn-on in current, upon the formation of multiple p-n junctions via the BPE array, suggests the conductance of the cell in the narrow BPE array region is significantly higher than the rest of the planar cell. In other words, the electronic conductance of the doped BPE region is much higher than the ionic conductance of the polymer electrolyte in the rest of the cell where the doping fronts have yet to meet.

The aluminum disc BPEs are not perfectly circular but elongated and tilted to the left. In fact, such a deformation is simply caused by a shadowing effect of the shadow mask that was not positioned directly above the evaporation source. Nevertheless, the BPEs are of similar sizes and p-doping from these BPEs only show a negligible variation in size. The length of the BPE in the direction of the applied electric field is about $0.3 \mathrm{~mm}$. For each BPE, the position of the p-doping front has been monitored as a function of time in Matlab. Figure 2 shows the p-doping tip position as a function of time averaged over all BPEs. The tip position was measured relative to the edge of the respective BPE. The error bars represent one standard deviation from the average value. Also shown is the average p-doping front position as a function of time in region L. Pdoping from both the BPE array and the driving electrode are linear functions of elapsed time. The slopes of the linear fits give the average p-doping propagation speeds as $0.27 \mathrm{~mm} \mathrm{~s}-1$ and $0.20 \mathrm{~mm} \mathrm{~s}-1$, respectively. The faster doping propagation speeds of the BPE doping is another significant key-observation that will be discussed below. 


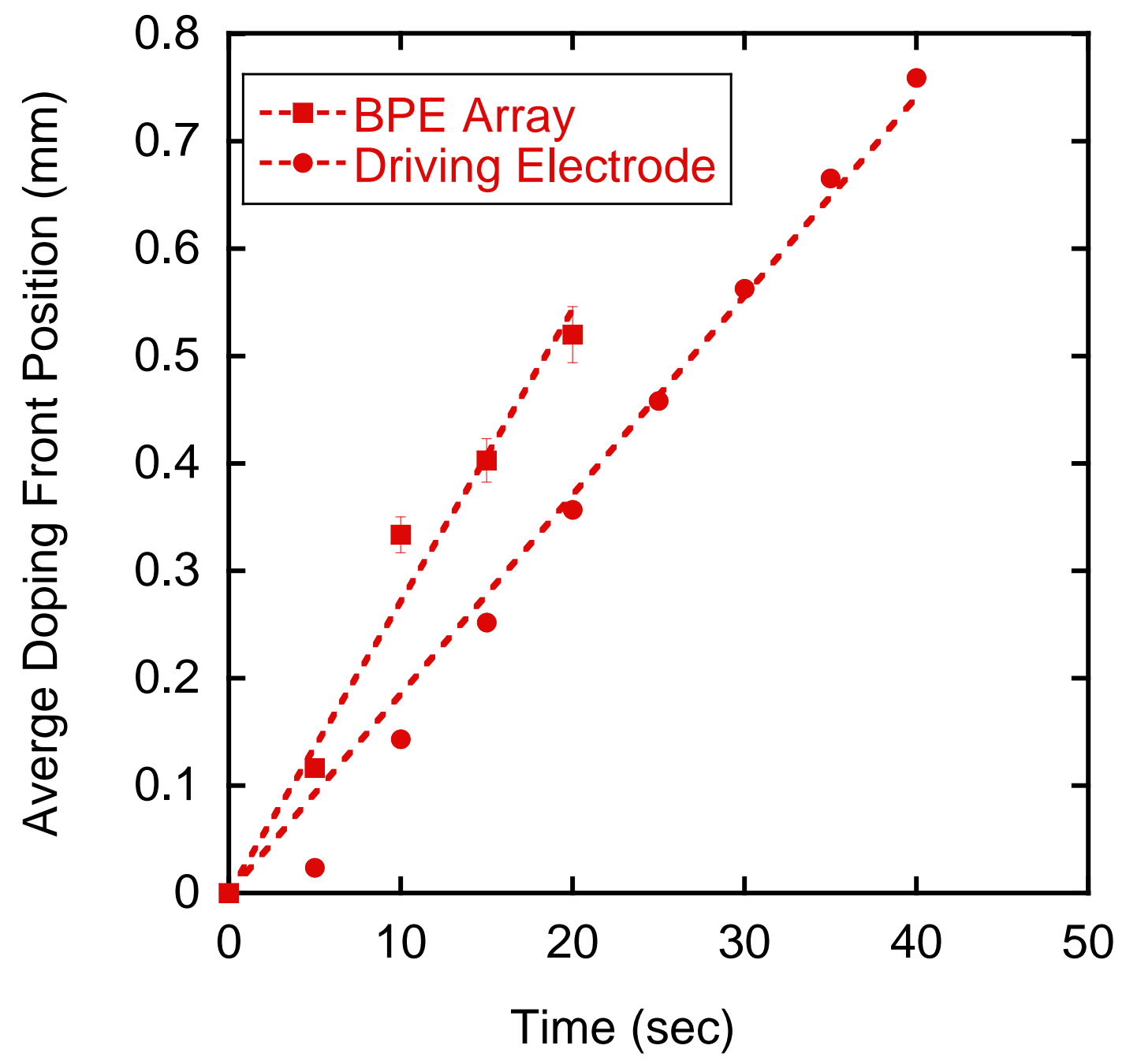

Figure 2. Cell 1-Average doping front position for p-doping originated from the BPE discs (square) and from the positive driving electrode (circle). For each BPE disc, the doping front position is calculated from the tip of the p-doping relative to the respective BPE disc. For the BPE array, the doping position is the average from the top seven BPE discs. The error bar represents one standard deviation. For p-doping from the driving electrode, the average is calculated in region L. The dashed lines are linear fits to the data. 
Figure 3 shows the time-lapse fluorescence images of a second cell (Cell 2) operated under identical conditions. The cell is nominally identical to the first cell although the BPE array is slightly shifted towards the cathode at the bottom. This cell exhibits much faster doping propagation. At $\mathrm{t}=15 \mathrm{~s}$, the $\mathrm{p}$ - and $\mathrm{n}$-doping from the BPEs have already made full contact. At $\mathrm{t}$ $=20 \mathrm{~s}$, EL is observed from all p-n junctions formed between the BPEs as well as between the BPE and the top or bottom driving electrode. The intensity of EL became stronger afterwards.

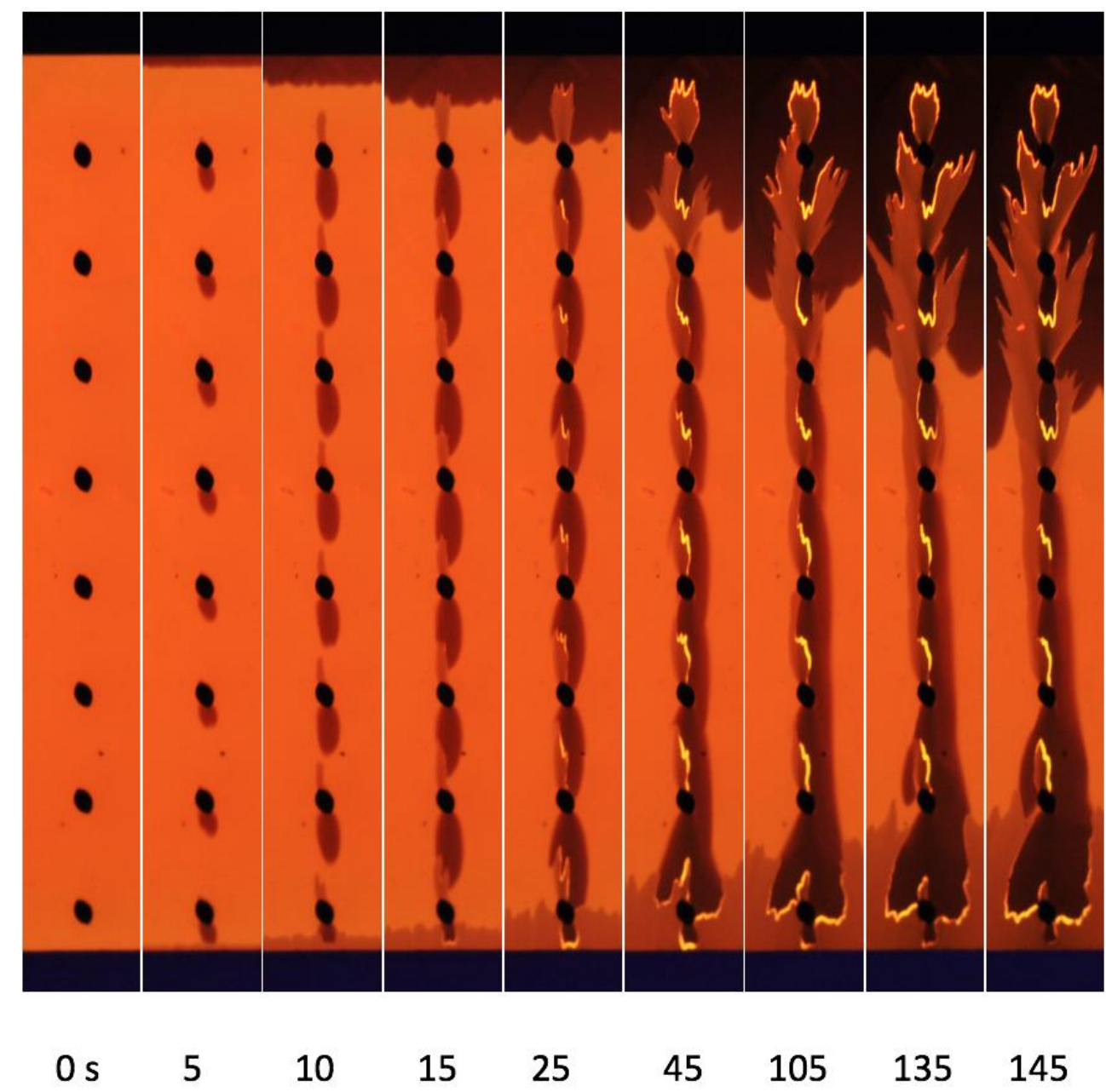

Figure 3. Cell 2-Time-lapsed fluorescence images of a second planar PLEC under $100 \mathrm{~V}$ bias and UV illumination. Only the region containing the BPE array is shown. The numbers underneath the images 
denote elapsed time in seconds. The bright lines between the BPE discs, visible after $25 \mathrm{~s}$ is due to p-n junction electroluminescence.

Figure 4 shows the current of Cell 2 as a function time. The peak current reached $244 \mu \mathrm{A}$ at $t=958 \mathrm{~s}$. By contrast, the peak current of Cell 1 was only $10.3 \mu \mathrm{A}$, reached at $\mathrm{t}=963 \mathrm{~s}$. The two cells were fabricated using the same casting solution and tested under identical conditions. A most likely cause of the large cell current difference is contact resistance arising from the pointlike contact between the metal probes and the soft and thin metal electrodes coated on top of the polymer film. A larger contact resistance in Cell 1 caused an effectively small bias voltage being applied between the driving electrodes. Despite the difference in absolute current levels, Cell 2 exhibits the same turn-on behavior as Cell 1 . At $\mathrm{t} \sim 300 \mathrm{~s}$, the turn-on represents the formation of a light-emitting $\mathrm{p}-\mathrm{n}$ junction between the $\mathrm{p}$ - and n-doping from the driving electrodes. This occurred much earlier than in Cell 1 due to the latter's faster doping propagation velocity. However, in that case, the doping propagation speed from the BPE discs is not calculated since only two images were captured before the formation of $p-n$ junctions. The inset of Figure 4 shows the current of Cell 2 in the first $50 \mathrm{~s}$. A significant turn-on is again observed. The two linear fits intersect at about $\mathrm{t}=15 \mathrm{~s}$, which is verified as the time when the $\mathrm{p}$ - and $\mathrm{n}$-doping from the BPEs have first made contact, as shown in Figure 3. Therefore, in both Cell 1 and Cell 2, the cell current underwent turn-ons at four different times that can all be explained by doping and the formation of light-emitting $\mathrm{p}-\mathrm{n}$ junctions. The earlier turn-ons originate from the formation of multiple p-n junctions in series via the BPEs. The latter turn-ons result from the junction formation in the rest of the planar LEC. 


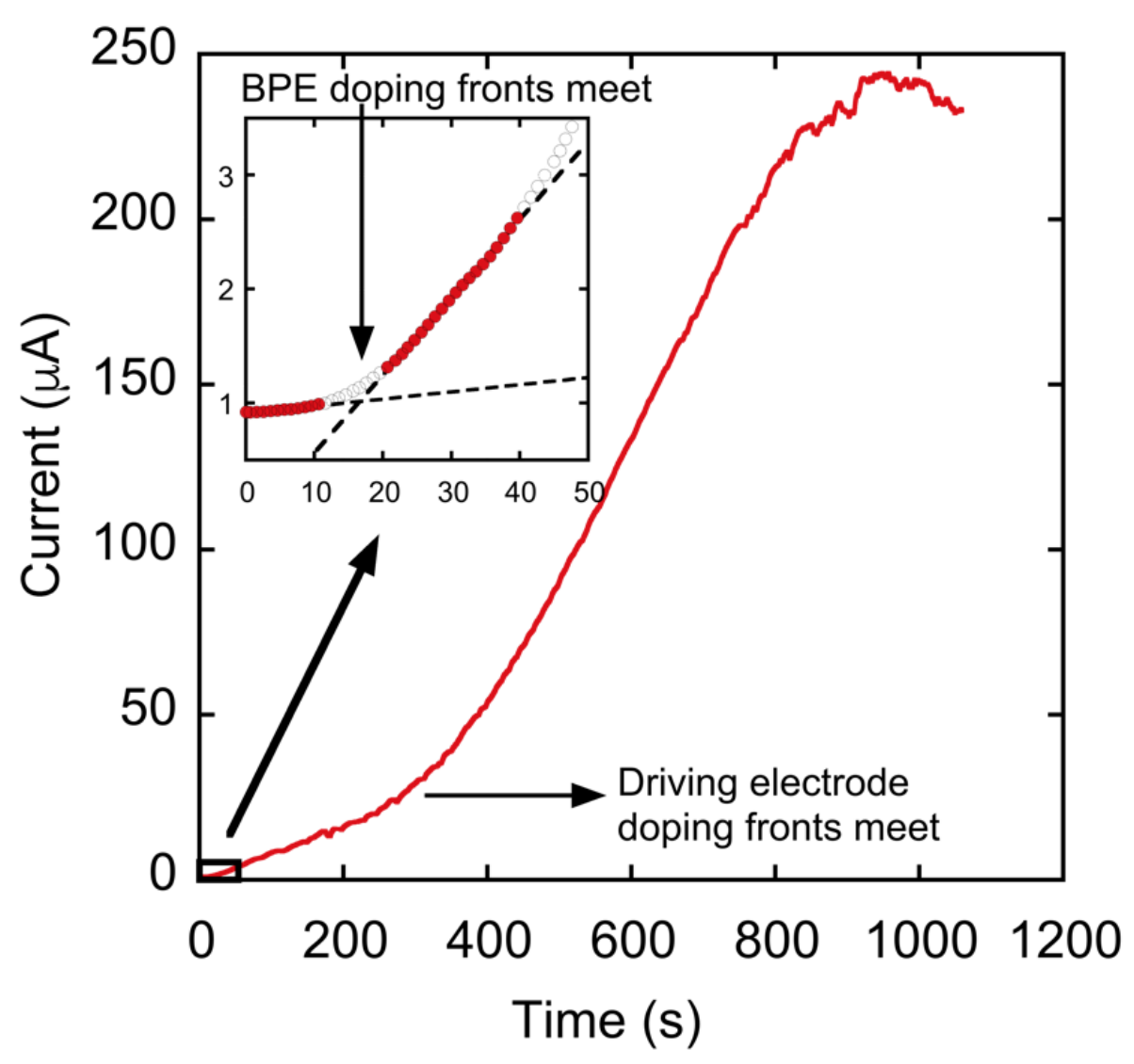

Figure 4. Cell 2-Cell current as a function of elapsed time after a $100 \mathrm{~V}$ bias voltage was applied to the gold (+) and aluminum (-) driving electrodes. The inset shows an expanded view of the cell current in the first $50 \mathrm{~s}$. The intersection between the linear fits performed to two segments of the curve determines an early current turn-on time at about $15 \mathrm{~s}$.

The doping patterns shown in Figure 3 have some distinct features. Initially, the BPE doping is narrow (about the width of the BPE discs) and confined between the BPEs. At $t>20 \mathrm{~s}$, doping at the top and bottom of the BPE array began to increase in size and branch out despite their contact with the opposite doping from the driving electrodes. Meanwhile, the light-emitting junctions between the middle BPEs (4-5 from the top) appear to be unaffected. In order to understand the doping patterns, COMSOL calculation was performed to map the electric field profile in the planar PLEC. As seen in Figure S1, the overall electric field profile of the planar PLEC, when the 
voltage bias was just applied to the driving electrodes, is that of a parallel plate capacitor. The presence of the BPE array has no effect on the field lines above and below the BPE array, at a distance larger than the diameter of the BPE disc. In the vicinity of the BPE array, however, the electric field is significantly distorted by the presence of the BPEs. The electric field is enhanced and the strongest at the top (cathode) and bottom (anode) extremities of BPE discs, and weakest at the left and right extremities of the BPE discs. The presence of BPEs also changed the direction of the electric field nearby, as indicated by the arrows in Figure S1. In a liquid electrochemical cell, the presence of metallic BPEs has been shown to alter the local electric field, leading to an observable effect called bipolar electrode focusing. $32-34$

The electric field profile shown in Figure S1 explains the key features of doping patterns along the BPE array. The initial p- and n-doping occurs preferentially at the top and bottom extremities of the BPE discs, where the potential difference between the metal BPE and the polymer film is the largest. This is due to both geometry and the aforementioned electric field enhancement. The latter is the cause of faster doping propagation at the BPEs compared to doping at the driving electrodes. The n-doping of Figure 1 (d), in particular, appears to have only occurred at the tips of the BPE cathodes. In Figure 3, a thicker n-doping indicates a larger potential difference at the BPE cathode surface to drive the reduction reaction. Once the localized doping reaction is initiated, the reaction fronts shift to the boundary between the conductive, doped polymer and the undoped polymer. The BPE p- and n-doping does not diverge as in the case of a single disc BPE in the open configuration.28 When the doping fronts meet to form p-n junctions, the outline of doped regions resembles the distorted field lines shown in Figure S1. We understand that doping cannot propagate in a direction perpendicular to the electric field because there is insufficient potential difference to drive the doping reactions. Regardless of the presence 
of a BPE or a BPE array, the direction of doping propagation is a visual indication of the direction of local electric field.

The BPE n-doping branched out when p-doping from the top driving electrode propagates pass the BPEs. This is at first counterintuitive since the BPE n-doping was enhanced, rather than suppressed by the opposite doping. The top of Figure 3 (i) shows that the BPE n-doping between the first three BPE discs exhibit sharp fingers pointed at a large angle away from the axis of the BPE array. The sharp fingers point to preferential directions of doping which again should align with the direction of electric field. The fact that the divergence of doping occurred near both ends of the BPE array suggests that the BPE array, as a whole, has become sufficiently conductive to function effectively as a single, rod-shaped BPE.

The polymer film between the BPEs became conductive when heavily doped $p-n$ junctions are formed between the individual BPE discs. These $p-n$ junctions are all under forward bias and connected in series by the BPE discs. The conductive BPE array did not short the PLEC, but did cause an early "turn-on" in cell current, as discussed earlier. It is well known that chemically doped polymers can exhibit metallic conductivities and are widely used in PLEDs and PLECs as hole-injection electrodes.35-38. Recently, we observe that electrochemically doped polymer in the interior of a planar PLEC exhibit doping patterns that can only be explained by treating the doped polymer as a bipolar electrode.39 In the current study, the polymers between the BPEs should have comparable or higher conductivity since de-doping of the polymer was prohibited. The BPE array, along with the doped polymer in between, is therefore a new type of BPE that is essentially a linear array of light-emitting p-n junctions connected (by the BPE discs) in series. The branching of the doping indicates the field lines have changed significantly from those of Figure S1, which is expected as soon as the BPE array adopts the shape of a conductive 
rod. Figure S2 shows COMSOL simulation of the cell with such a rod-shaped BPE. In the simulation, the doped regions between the BPE discs are made conductive by introducing a complex permittivity with a large imaginary component. The field lines are dramatically different from those of Figure S1. The distortion of field lines occurred over a much larger distance around the BPE array. In particular, the field lines near the ends of the rod are now at a large angle relative to the rod. This large divergence of the field lines is mainly responsible for the divergence of doping.

\section{Conclusions}

In summary, extremely large, planar PLECs containing a metal BPE array in the open configuration have been studied. The planar PLECs are driven by a constant bias voltage of 100 $\mathrm{V}$ applied between the planar driving electrodes. This caused in situ electrochemical doping of the luminescent polymer that is visible as fluorescence-quenched regions under UV illumination. Time-lapse fluorescence imaging of the PLEC under operation reveals that opposite doping originated from neighboring BPE discs can indeed interact to form multiple light-emitting $\mathrm{p}-\mathrm{n}$ junctions in series. This provides the first direct evidence of the operation mechanism of bulk homojunction PLECs containing dispersed metallic particles. P-doping propagation from BPE discs is faster than that of p-doping from the flat driving electrodes due to electric field enhancement at the extremities of the BPE electrodes. The near simultaneous formation of multiple light-emitting $\mathrm{p}-\mathrm{n}$ junctions in series causes a sharp increase in cell current. This indicates that the region containing the BPE is much more conductive than the rest of the planar cell despite the latter's greater width. The p- and n-doping originated from the BPE discs are initially highly confined. Significant expansion and divergence of doping occurred when the region containing the BPE array became more conductive. The shape and direction of expanded 
doping strongly suggests that the multiple light-emitting p-n junctions, formed in between and connected by the array of metal BPEs, have functioned as a single rod-shaped BPE. This represents a new type of BPE that is formed in situ and a combination of metal, doped polymers and forward-biased p-n junctions connected in series.

Corresponding Author

* Prof. Jun Gao

Email: jungao@queensu.ca

\section{ACKNOWLEDGMENT}

The study at Queen's University was supported by the Natural Sciences and Engineering Research Council of Canada (NSERC). L.B. and G.W. acknowledge the University of Bordeaux and the Centre National de la Recherche Scientifique for financial support to exploratory research (PEPS IdEx/CNRS). 


\section{Supplemental Information}

\section{Bipolar Electrode Array Embedded in a Polymer Light-Emitting Electrochemical Cell}

Jun Gao1, Shulun Chenı, Faleh AlTal1, Shiyu Hu1, Laurent Bouffier2 and Guillaume Wantz3

1Department of Physics, Engineering Physics and Astronomy, Queen's University, Kingston, Ontario, K7L 3N6, Canada

2Univ. Bordeaux, ISM, CNRS, UMR 5255, Bordeaux INP, F-33400 Talence, France

3Univ. Bordeaux, IMS, CNRS, UMR 5218, Bordeaux INP, F-33405 Talence, France

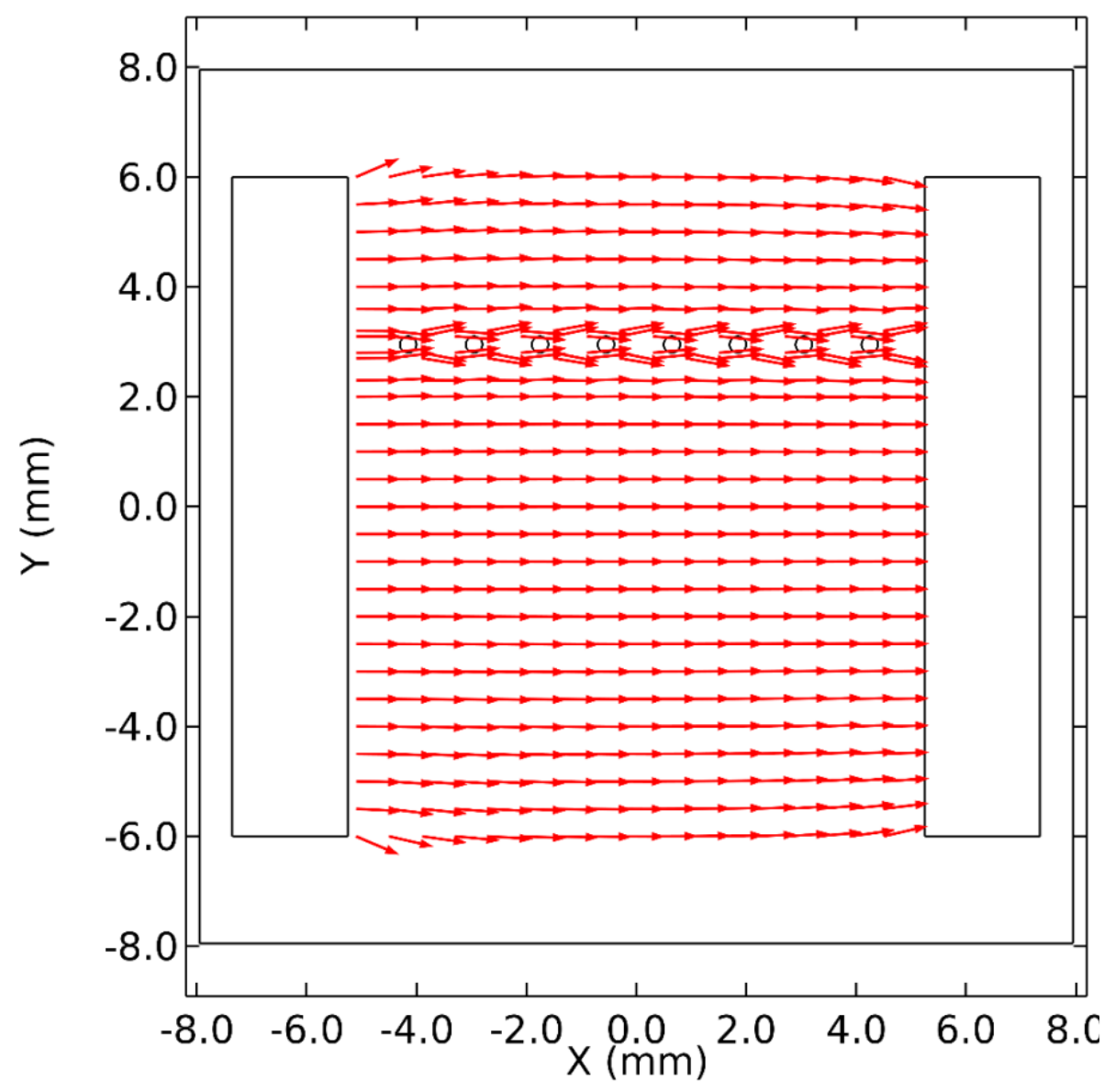


Figure S1. COMSOL calculation of electric field distribution for a planar PLEC with an embedded BPE array. The electric field was calculated by solving the equation: $\nabla\left({ }_{0}, \nabla V\right)=$ and $E=\nabla V$; where $\varepsilon 0$ is the vacuum permittivity, $\varepsilon r$ is the relative permittivity, $V$ is the electrostatic potential, $\rho$ is the charge density, and $E$ is the electric field vector. The value of relative permittivity was set to 3 for the pristine polymer film. The potentials were set to $0 \mathrm{~V}$ and $100 \mathrm{~V}$, respectively, at the driving electrodes. Some detailed features of the actual cells in Figure 1 and Figure 3 were not considered. These include, for example, the rounding of the corners of the driving electrodes and the deviation of the BPEs from perfect circles. For clarity, the length of the field arrows was logarithmically scaled relative to the natural logarithm of the electric field at the base of the arrow. The graph is for qualitative illustration only and when the bias voltage has just been applied to the driving electrodes. 


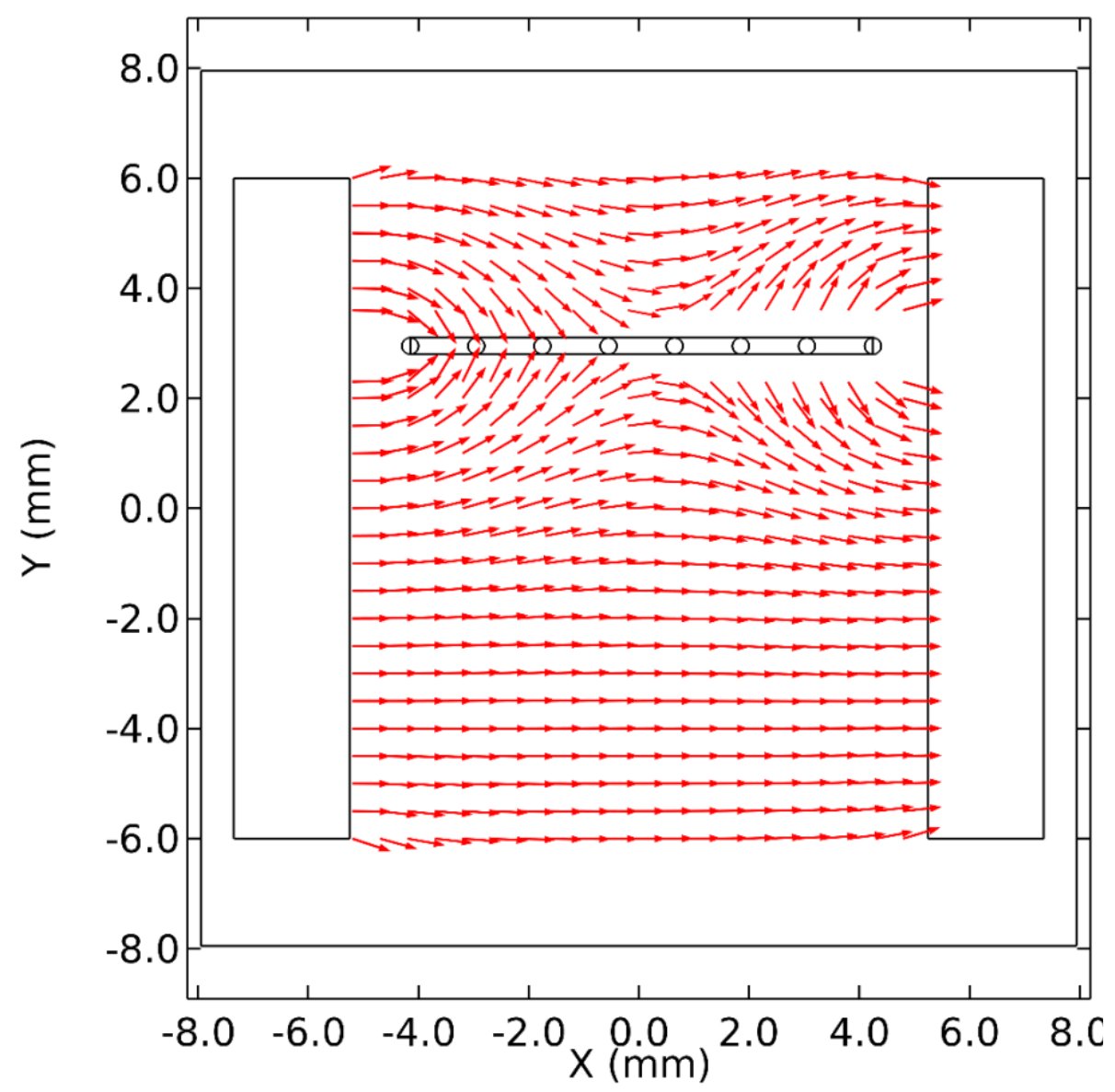

Figure S2. COMSOL calculation of electric field for a planar PLEC with an embedded BPE array. Complex permittivity values were used for the BPE discs $(1-\mathrm{j} 2500)$ and the doped polymer between the BPEs (3-j250). These values were chosen for qualitative illustration of the field profile after the formation of light-emitting p-n junctions between the BPEs. 


\section{REFERENCES}

1. Sentic, M.; Arbault, S.; Bouffier, L.; Manojlovic, D.; Kuhn, A.; Sojic, N., 3d Electrogenerated Chemiluminescence: From Surface-Confined Reactions to Bulk Emission. Chem. Sci. 2015, 6, 44334437.

2. de Poulpiquet, A.; Diez-Buitrago, B.; Milutinovic, M.; Goudeau, B.; Bouffier, L.; Arbault, S.; Kuhn, A.; Sojic, N., Dual-Color Electrogenerated Chemiluminescence from Dispersions of Conductive Microbeads Addressed by Bipolar Electrochemistry. ChemElectroChem 2016, 3, 404-409.

3. $\quad$ Loget, G.; Roche, J.; Kuhn, A., True Bulk Synthesis of Janus Objects by Bipolar Electrochemistry. Advanced Materials 2012, 24, 5111-5116.

4. $\quad$ Loget, G.; Lapeyre, V.; Garrigue, P.; Warakulwit, C.; Limtrakul, J.; Delville, M.-H.; Kuhn, A., Versatile Procedure for Synthesis of Janus-Type Carbon Tubes. Chemistry of Materials 2011, 23, 25952599.

5. $\quad$ Bouffier, L.; Ravaine, V.; Sojic, N.; Kuhn, A., Electric Fields for Generating Unconventional Motion of Small Objects. Current Opinion in Colloid \& Interface Science 2016, 21, 57-64.

6. Loget, G.; Kuhn, A., Shaping and Exploring the Micro- and Nanoworld Using Bipolar Electrochemistry. Anal. Bioanal. Chem. 2011, 400, 1691-1704.

7. Loget, G.; Zigah, D.; Bouffier, L.; Sojic, N.; Kuhn, A., Bipolar Electrochemistry: From Materials Science to Motion and Beyond. Accounts of Chemical Research 2013, 46, 2513-2523. 8. $\quad$ Fosdick, S. E.; Knust, K. N.; Scida, K.; Crooks, R. M., Bipolar Electrochemistry. Angewandte Chemie-International Edition 2013, 52, 10438-10456.

9. Chow, K.-F.; Mavré, F.; Crooks, R. M., Wireless Electrochemical DNA Microarray Sensor. Journal of the American Chemical Society 2008, 130, 7544-+.

10. Inagi, S., Fabrication of Gradient Polymer Surfaces Using Bipolar Electrochemistry. Polymer Journal 2016, 48, 39-44.

11. Tracy, C.; Gao, J., Polymer Bulk Homojunction Photonic Devices. Applied Physics Letters 2005, 87, 143502 .

12. Tracy, C.; Gao, J., Polymer Bulk Homojunction Light-Emitting Electrochemical Cells. Journal of Applied Physics 2006, 100, 104503.

13. Pei, Q. B.; Yu, G.; Zhang, C.; Yang, Y.; Heeger, A. J., Polymer Light-Emitting ElectrochemicalCells. Science 1995, 269, 1086-1088.

14. Tang, S.; Edman, L., Light-Emitting Electrochemical Cells: A Review on Recent Progress. Topics in Current Chemistry 2016, 374.

15. Xiong, Y.; Li, L.; Liang, J. J.; Gao, H.; Chou, S. Y.; Pei, Q. B., Efficient White Polymer LightEmitting Electrochemical Cells. Materials Horizons 2015, 2, 338-343.

16. Ayguler, M. F.; Weber, M. D.; Puscher, B. M. D.; Medina, D. D.; Docampo, P.; Costa, R. D., Light-Emitting Electrochemical Cells Based on Hybrid Lead Halide Perovskite Nanoparticles. Journal of Physical Chemistry C 2015, 119, 12047-12054.

17. Costa, R. D.; Ortí, E.; Bolink, H. J.; Monti, F.; Accorsi, G.; Armaroli, N., Luminescent Ionic Transition-Metal Complexes for Light-Emitting Electrochemical Cells. Angew. Chem. Int. Ed. 2012, 51, 8178-8211.

18. Su, H. C.; Cheng, C. Y., Recent Advances in Solid-State White Light-Emitting Electrochemical Cells. Israel Journal of Chemistry 2014, 54, 855-866.

19. Meier, S. B.; Tordera, D.; Pertegas, A.; Roldan-Carmona, C.; Orti, E.; Bolink, H. J., LightEmitting Electrochemical Cells: Recent Progress and Future Prospects. Materials Today 2014, 17, 217223.

20. Xiao, Y.; Xu, L. R.; Qi, L. W., Electrochemiluminescence Bipolar Electrode Array for the Multiplexed Detection of Glucose, Lactate and Choline Based on a Versatile Enzymatic Approach. Talanta 2017, 165, 577-583. 
21. Khoshfetrat, S. M.; Ranjbari, M.; Shayan, M.; Mehrgardi, M. A.; Kiani, A., Wireless Electrochemiluminescence Bipolar Electrode Array for Visualized Genotyping of Single Nucleotide Polymorphism. Anal. Chem. 2015, 87, 8123-8131.

22. Zhai, Q. F.; Zhang, X. W.; Han, Y. C.; Zhai, J. F.; Li, J.; Wang, E. K., A Nanoscale Multichannel Closed Bipolar Electrode Array for Electrochemiluminescence Sensing Platform. Anal. Chem. 2016, 88, 945-951.

23. Termebaf, H.; Shayan, M.; Kiani, A., Two-Step Bipolar Electrochemistry: Generation of Composition Gradient and Visual Screening of Electrocatalytic Activity. Langmuir 2015, 31, 1323813246.

24. Lin, X.; Zheng, L.; Gao, G.; Chi, Y.; Chen, G., Electrochemiluminescence Imaging-Based HighThroughput Screening Platform for Electrocatalysts Used in Fuel Cells. Anal. Chem. 2012, 84, 77007707.

25. Fosdick, S. E.; Berglund, S. P.; Mullins, C. B.; Crooks, R. M., Parallel Screening of Electrocatalyst Candidates Using Bipolar Electrochemistry. Anal. Chem. 2013, 85, 2493-2499.

26. Chow, K.-F.; Mavré, F.; Crooks, J. A.; Chang, B.-Y.; Crooks, R. M., A Large-Scale, Wireless Electrochemical Bipolar Electrode Microarray. Journal of the American Chemical Society 2009, 131, 8364-+.

27. Koefoed, L.; Pedersen, E. B.; Thyssen, L.; Vinther, J.; Kristiansen, T.; Pedersen, S. U.; Daasbjerg, K., Functionalizing Arrays of Transferred Monolayer Graphene on Insulating Surfaces by Bipolar Electrochemistry. Langmuir 2016, 32, 6289-6296.

28. Chen, S. L.; Wantz, G.; Bouffier, L.; Gao, J., Solid-State Bipolar Electrochemistry: PolymerBased Light-Emitting Electrochemical Cells. ChemElectroChem 2016, 3, 392-398.

29. Hu, Y. F.; Tracy, C.; Gao, J., High-Resolution Imaging of Electrochemical Doping and Dedoping Processes in Luminescent Conjugated Polymers. Applied Physics Letters 2006, 88, 123507.

30. Alem, S.; Gao, J., The Effect of Annealing/Quenching on the Performance of Polymer LightEmitting Electrochemical Cells. Organic Electronics 2008, 9, 347-354.

31. Hohertz, D.; Gao, J., How Electrode Work Function Affects Doping and Electroluminescence of Polymer Light-Emitting Electrochemical Cells. Advanced Materials 2008, 20, 3298-3302.

32. Dhopeshwarkar, R.; Hlushkou, D.; Nguyen, M.; Tallarek, U.; Crooks, R. M., Electrokinetics in Microfluidic Channels Containing a Floating Electrode. Journal of the American Chemical Society 2008, 130, 10480-10481.

33. Hlushkou, D.; Perdue, R. K.; Dhopeshwarkar, R.; Crooks, R. M.; Tallarek, U., Electric Field Gradient Focusing in Microchannels with Embedded Bipolar Electrode. Lab on a Chip 2009, 9, 1903 1913.

34. Anand, R. K.; Sheridan, E.; Hlushkou, D.; Tallarek, U.; Crooks, R. M., Bipolar Electrode Focusing: Tuning the Electric Field Gradient. Lab on a Chip 2011, 11, 518-527.

35. Matyba, P.; Yamaguchi, H.; Chhowalla, M.; Robinson, N. D.; Edman, L., Flexible and MetalFree Light-Emitting Electrochemical Cells Based on Graphene and Pedot-Pss as the Electrode Materials. ACS Nano 2011, 5, 574-580.

36. Gao, J.; Heeger, A. J.; Lee, J. Y.; Kim, C. Y., Soluble Polypyrrole as the Transparent Anode in Polymer Light-Emitting Diodes. Synthetic Metals 1996, 82, 221-223.

37. Cao, Y.; Yu, G.; Zhang, C.; Menon, R.; Heeger, A. J., Polymer Light-Emitting Diodes with Polyethylene Dioxythiophene-Polystyrene Sulfonate as the Transparent Anode. Synthetic Metals 1997, 87, 171-174.

38. Yang, Y.; Westerweele, E.; Zhang, C.; Smith, P.; Heeger, A. J., Enhanced Performance of Polymer Light-Emitting-Diodes Using High-Surface-Area Polyaniline Network Electrodes. Journal of Applied Physics 1995, 77, 694-698.

39. Hu, S. Y.; Chi, X.; Chen, S. L.; AlTal, F.; Gao, J., Visualizing the Bipolar Electrochemistry of Electrochemically Doped Luminescent Conjugated Polymers. Journal of Physical Chemistry C 2017, $121,8409-8415$. 
